\title{
Growth promoting and therapeutic antibiotics affect chicken microbiomes and resistomes
}

Chhedi Lal Gupta

Shlomo E. Blum

Karuppasamy Kattusamy

Tali Daniel

Shelly Druyan

Roni Shapira

Oleg Krifucks

Yong-Guan Zhu

Xin-Yuan Zhou

Jian-Qiang Su

Eddie Cytryn

\section{Video Byte}

Keywords: Microbiome, broiler chickens, poultry, growth promoting antibiotics, shotgun metagenomics, antimicrobial resistance, antibiotic resistant bacteria, antibiotic resistance genes, priority pathogens, $16 \mathrm{~S}$ rRNA gene amplicon sequencing, HT-qPCR, antibiotics, antimicrobials

Posted Date: October 13th, 2021

DOl: https://doi.org/10.21203/rs.3.rs-967525/v1

License: (a) (1) This work is licensed under a Creative Commons Attribution 4.0 International License.

Read Full License 


\section{Abstract}

Antibiotics are commonly used as both therapeutics and growth promoters in the poultry industry. Antibiotic use in animal husbandry has been linked to proliferation of antimicrobial resistance, but current evidence is indirect. To examine this problem directly, researchers studied the impact of the growth promoter bacitracin and the therapeutic antibiotic enrofloxacin on microbiomes and resistomes. They sampled both cloacal swabs and litter as proxies of gut and environmentally-disseminated microbiomes, coupling standard isolation and metagenomic methodologies. Antibiotic-resistant bacteria and resistance genes were ubiquitous in both the gut and litter and most of the variation in the microbiomes and resistomes was attributable to either growth stage or sample source. But, bacitracin-fed birds had higher levels of bacitracin resistance genes, and a greater proportion of their Enterococcaceae population was vancomycin-resistant. The researchers also found that, while many of the Enterococcaceae strains were human-associated, the vancomycin-resistant strains were nearly exclusively avian-associated, suggesting that resistant versus non-resistant strains have different taxonomic profiles. Enrofloxacin had highly variable effects but generally increased the abundance of multidrug-resistant Enterobacteriaceae. These results demonstrate that while antibiotic use can influence microbiomes and resistomes in a complex manner, this kind of antibiotic use may select for antimicrobial resistance. 